U.S. Department of Commerce-Bureau of Standards

RESEARCH PAPER RP576

Part of Bureau of Standards Journal of Research, Vol. 11, July 1933

\title{
A 200-KILOCYCLE PIEZO OSCILLATOR
}

\author{
By E. G. Lapham
}

ABSTRACT

This paper describes a piezo oscillator which is used to control the frequency of the standard-frequency transmissions of the Bureau. The unit incorporates in its design a double temperature control of the quartz plate, temperature control of the oscillator and amplifier, and a clamped type of quartz-plate mounting.

\section{CONTENTS}

I. Introduction

II. Initial tests

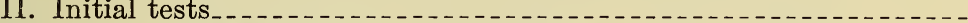

III. General arrangement

IV. Quartz plate and holder

V. Circuit arrangement.

VI. Temperature control

VII. Results

VIII. Acknowledgment.

\section{INTRODUCTION}

This paper describes a piezo oscillator specially designed for constancy over periods of a few hours. It is used in controlling the frequency of the radio transmitter used in the Bureau's weekly standard-frequency transmissions. The transmissions are for 2 hours at a time, on a frequency of 5,000 kilocycles per second, and it is required that the accuracy be maintained at all times better than 1 cycle per second ( 1 part in 5,000,000). This imposes rigorous requirements upon the piezo oscillator used to control the frequency of the radio transmitter.

\section{INITIAL TESTS}

The piezo oscillators which were available at the beginning of the 5,000-kilocycle transmissions were used for several months. The frequency of the radio transmitter was constant to approximately 1 part in 1,000,000. Careful checking between the frequency monitor and the transmitter revealed three main sources of variation. The first was a transient effect which was caused by the reaction of the radio transmitter on the piezo oscillator. The magnitude of this variation was indeterminate because of its short duration. The second source of variation was the operation of the heater relays. This caused an abrupt frequency change of the order of 5 to 8 parts in $10^{8}$. The third variation was a slow drift in the temperature of the quartz plate due to changes in room temperature, which caused the frequency to change at the rate of approximately 1 part in $10^{6}$ per hour. This rate of variation would not continue indefinitely but it might continue for several hours. 
The reaction of the radio transmitter on the piezo oscillator was noticeable immediately after keying the transmitter. While the transmitter was being keyed the plate current in the oscillator circuit of the piezo oscillator would vary in synchronism with the keying. At the termination of the keying a short time would elapse before the frequency of the standard reached its previous steady condition. The most evident solution was to improve the shielding around the standard and to further decrease the coupling between the amplifier and the oscillator.

The operation of the heater relays in the temperature control of the piezo oscillator was also found to produce an abrupt change in frequency in certain of the standards tested. The change in frequency was too abrupt to be due to any cyclic variation in temperature which might occur as the heat was applied. It was found to be due to a change in the capacity of the plate holder with respect to ground. This variation was found to be eliminated by proper grounding of the metal boxes surrounding the heaters and careful shielding of leads which passed through the heater compartment. These precautions were also effective in reducing the a.c. modulation which is often induced by the heaters when operated on alternating current.

The frequency variation which was most important was that due to a drift in the temperature of the quartz plate. The radio transmitter was at the time located in a temporary building. The room temperature varied between $15^{\circ}$ and $35^{\circ} \mathrm{C}$. The quartz plates which are used in the secondary standards have a temperature coefficient of frequency of approximately -20 parts in $10^{6}$ per ${ }^{\circ} \mathrm{C}$. A change in the temperature of the quartz plate of $0.01^{\circ} \mathrm{C}$. produces a change in frequency of 1 cycle in 5,000 kilocycles, which is the maximum tolerance. The single temperature controls with which the available secondary standards were equipped were not sufficiently constant when subjected to such wide variations in room temperature. There were three possible courses, any or all of which could be used to maintain a more constant frequency-(1) provide a quartz plate with a lower temperature coefficient of frequency, (2) provide additional temperature control for the piezo oscillator, and (3) provide a variable condenser to compensate for unavoidable temperature changes. The two latter courses were adopted in the design of a new piezo oscillator. A second temperature-controlled compartment surrounding the quartz plate and oscillator and amplifier circuit arrangements decreased the temperature variations so that only a very narrow range of frequency adjustment was required by (3)

\section{GENERAL ARRANGEMENT OF PIEZO OSCILLATOR}

The general arrangement of the piezo oscillator is shown in figures 1 and 2. Figure 1 is an exterior view. The filament and plate voltmeters and the oscillator plate-current meter are at the right. The two heater indicator lamps are located on the left-hand side of the panel. In the lower center the end of a slotted shaft is visible which is for small frequency adjustments. Guards for the two thermometers are on the top. The filament and plate batteries are mounted in a metal box, and connecting leads pass through a flexible metal sheath. The B batteries were later replaced by a rectifier. 


\section{B.S. Journal of Research, RP576}

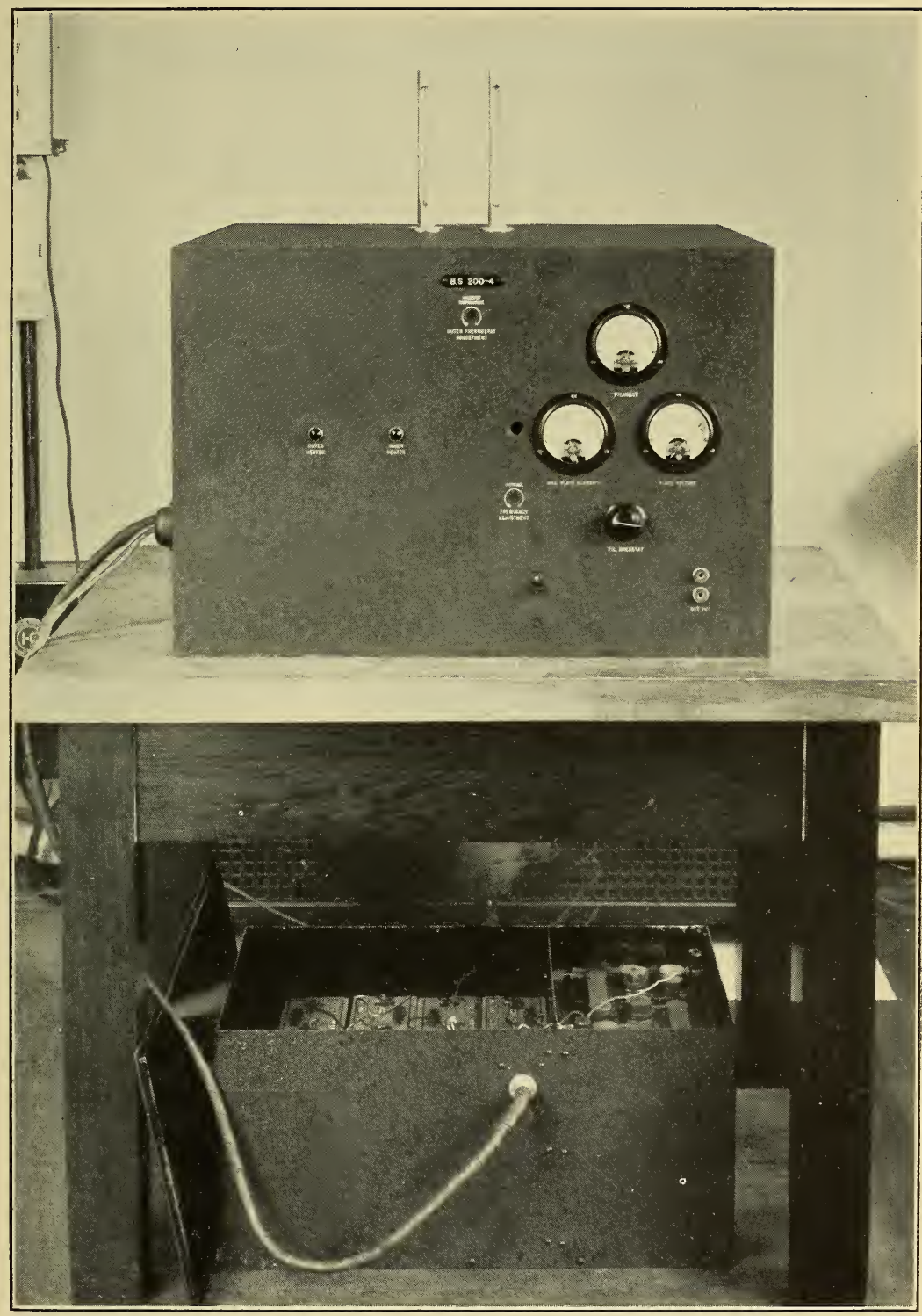

FiguRe 1.-Front view of piezo oscillator and shielded battery compartment. 
B.S. Journal of Research, RP576

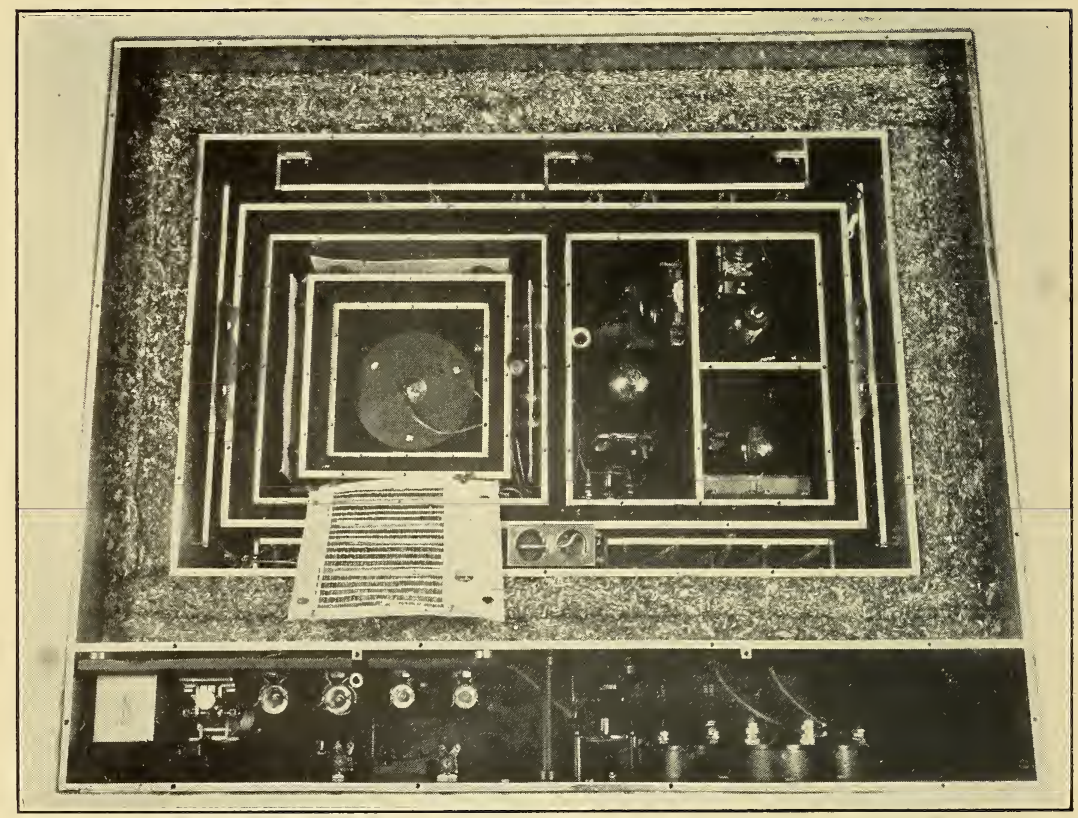

Figure 2.-Interior view of piezo oscillator. 
The plate voltage on the oscillator tube was maintained at 90 volts by means of a UX874 voltage regulator tube. Figure 2 is an interior view. A 4-inch space between the front panel and the temperaturecontrolled compartment is provided in which the heater control equipment, terminal strip, and meters are located. The temperature control is divided into two parts. The oscillator and amplifier circuit arrangements are in the section at the right and the quartz plate is in the section at the left. The bimetallic thermostat in this view was later replaced by a mercury thermostat. The bimetallic thermostat was not sufficiently sensitive to be satisfactory for the outer temperature control.

\section{QUARTZ PLATE AND HOLDER}

The piezo-electric element is a Curie-cut or zero-cut quartz plate. It is circular in shape and the dimensions are such that the fundamental frequency is 200 kilocycles per second at approximately $45^{\circ} \mathrm{C}$. The quartz plate is held in position between the electrodes ${ }^{1}$ by means of three screws which bear at the bottom of a V-shaped.

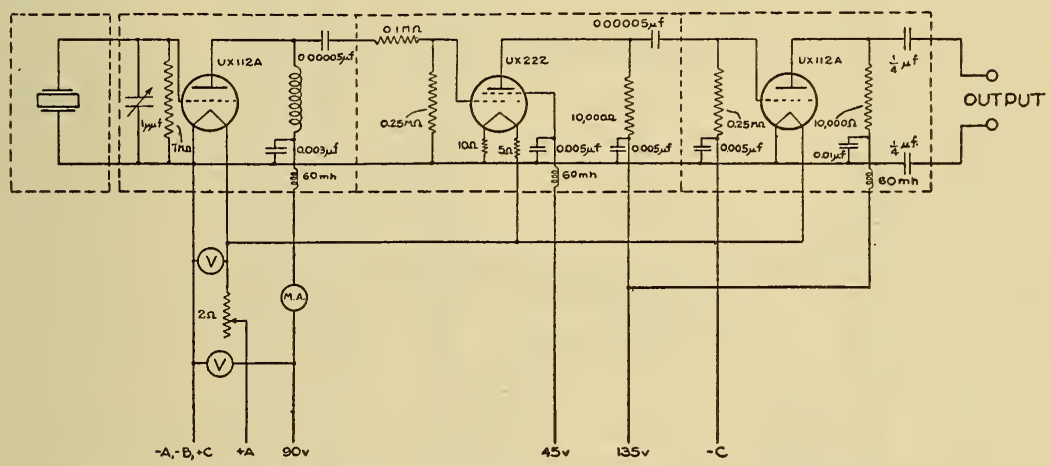

FIgURE 3.-Oscillator and amplifier circuit arrangement.

groove on the cylindrical surface of the plate midway between the plane faces.

\section{CIRCUIT ARRANGEMENT}

The circuit arrangement (fig. 3) is essentially the same as ${ }^{\mathrm{T}}$ that used in previous secondary standards. ${ }^{2}$ The quartz plate is connected between grid and filament of a UX112A tube and an untuned inductance is used as the plate impedance. The coupling to the first amplifier, a UX222 tube, is reduced considerably by the voltage divider arrangement indicated in the figure. This coupling amplifier is followed by another stage using a UX112A tube and straight capacity-resistance coupling. The manual frequency control consists of a 2-plate variable condenser connected in parallel with the quartz plate. The maximum capacity of this condenser is approximately $2 \mu \mu \mathrm{f}$. A very gradual adjustment of the frequency is provided by means of a 60 to 1 reduction through a friction drive and a set of gears.

1 V. E. Heaton and E. G. Lapham, Quartz Plate Mountings and Temperature Control for Piezo Oscillators, Proc. I.R.E., vol. 20, p. 261, February 1932. B.S. Jour. Research, vol. 7 (RP366) October 1931.

2 V. E. Heaton and W. H. Brattain, Design of a Portable Temperature-controlled Piezo Oscillator, Proc.

I.R.E., vol. 18, p. 345, July 1930. B. S. Jour. Research, vol. 4 (RP153), March 1930. 


\section{TEMPERATURE CONTROL}

Figure 4 is a schematic diagram of the temperature control. It consists of two thermostatically-controlled compartments, one within the other. The outer compartment is operated at a temperature about $1.5^{\circ}$ lower than the inner one. The quartz plate is located within the inner compartment so that it has a double temperature control. The oscillator and amplifier circuit arrangements are placed

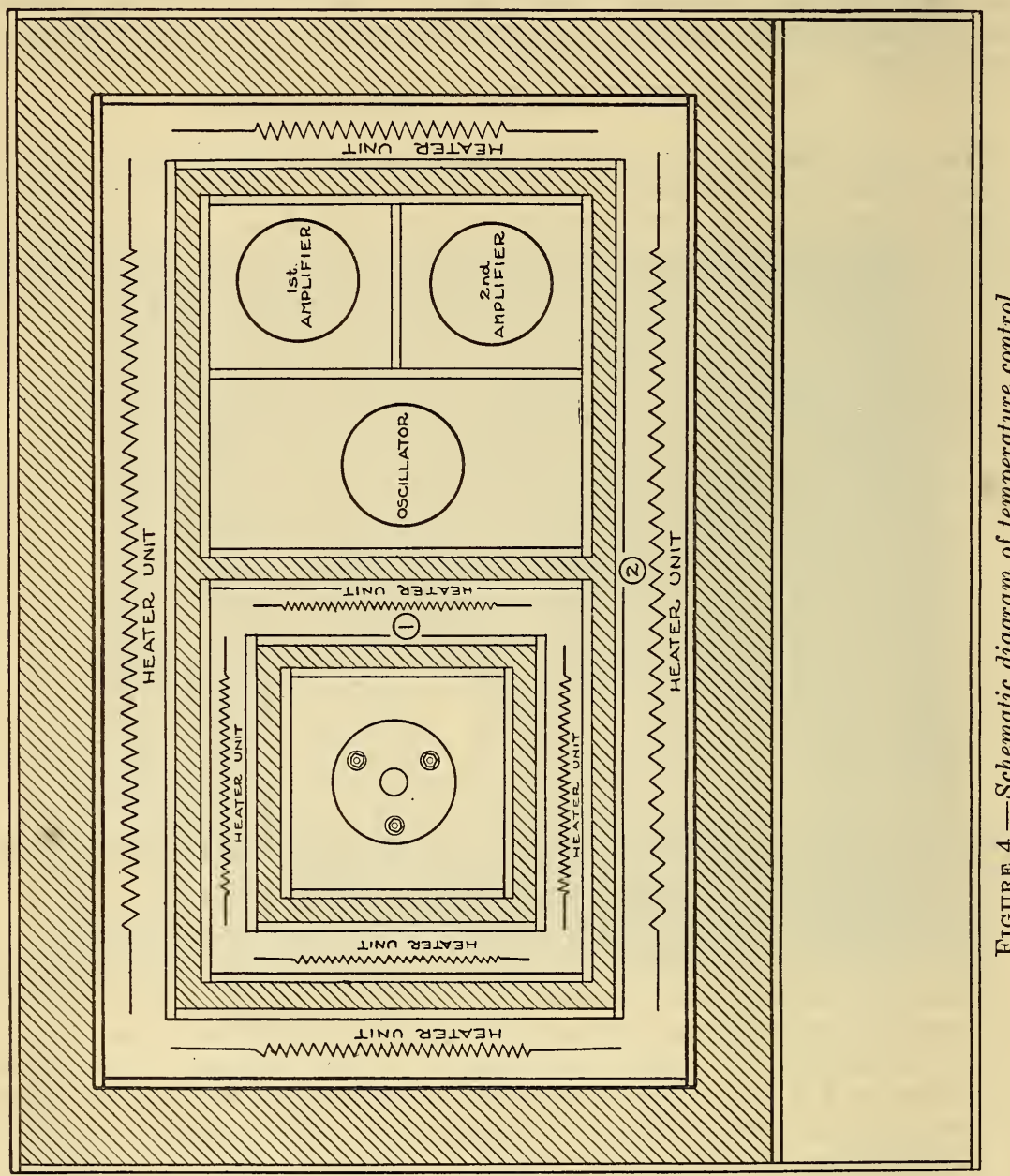

within the outer temperature control only. Two cubical boxes, constructed of $3 / 16$-inch aluminum, serve to equalize the temperature on all sides of the quartz plate. The two boxes are separated by three layers of $1 / 4$-inch felt, as indicated by the diagonal lines, which reduce the rate of flow of heat through the walls. The inner heaters are 1-ampere heater grids, one mounted on each wall of the temperaturecontrolled space. A 5-inch mercury thermostat is used to control the temperature. It is mounted vertically on the inner wall of the compartment between the heater and the heat-attenuating layers, in 
the position indicated by the numeral 1 in the diagram. Additional layers of felt and another aluminum box separate this unit and the one containing the oscillator circuit arrangement from the outer heaters. The outer heaters are constructed of no. 28 nichrome wire wound into a spiral which is looped back and forth across an insulating framework. The temperature of this outer space is likewise controlled by a mercury thermostat which is mounted in an aluminum well in the position indicated by the numeral 2 . The extreme outer wall is composed of two thicknesses of $3 / 4$-inch hair-felt enclosed by a $3 / 16$-inch aluminum box.

The heater control circuits are shown in figure 5. The relays are of the sensitive polarized type which operate very positively on 4 milliamperes direct current. The necessary voltage to operate the relays is furnished by a step-down transformer and a copper-oxide

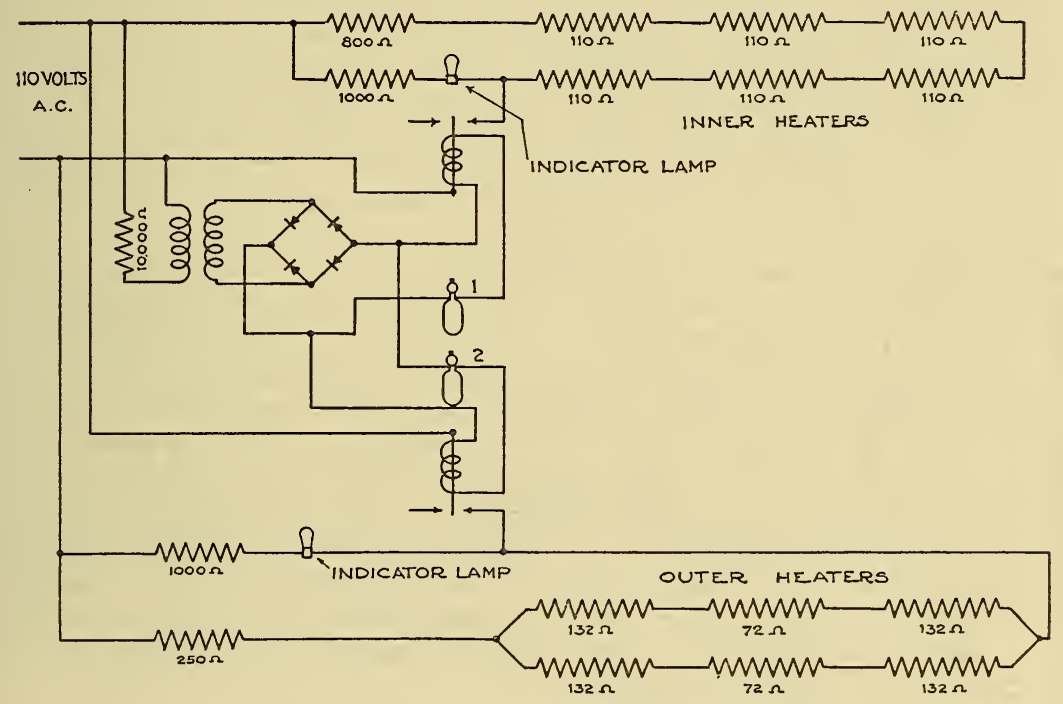

Figure 5.-Temperature-control circuit arrangement.

rectifier. The contacts on the relays were replaced by tungsten contacts so as to prevent burning and sticking.

The use of this piezo oscillator has shown one major limitation. The temperature of the quartz plate is slightly higher when the tubes are operating than when they are not. This fact makes it necessary to turn the oscillator on at least 24 hours before it is to be used in order to keep the frequency highly constant. The change in frequency due to the heat from the tubes is approximately 2 parts in $1,000,000$. There are various ways in which this effect could be minimized. One would be to increase the thickness of felt between the tube compartment and the quartz plate compartment and decrease the heat attenuation between the tube compartment and the outer heater. The result would be that the heat from the tubes would be dissipated more rapidly to the outer temperature-controlled space and result in a lower temperature of the tube compartment, which would, accordingly, reduce the effect on the temperature of the quartz plate. 
Several holes through the top of the box surrounding the tubes would also be effective in reducing the temperature effect upon the quartz plate. Another solution would be to have fixed heaters located in the compartment containing the tubes, and adjusted to furnish an amount of energy equal to that dissipated by them. The heaters could then be connected to the switch operating the tube filaments, so that when filaments were off the heaters would be on. The life of the tubes could thus be conserved without disturbing the temperature regulation.

\section{RESULTS}

The piezo oscillator was completed in August 1931. During the first three months that it was in use to control the standard-frequency transmissions, the frequency drifted to a lower value so that several temperature adjustments were required in order to maintain the correct frequency. Such a frequency drift seems to be the normal behavior with this clamped type of holder. On December 1, 1931, a temperature adjustment was made and new tubes installed. For one year from that date this piezo oscillator controlled the standardfrequency transmitter each week without any further adjustments, except, of course, for slight changes in the condenser for fine frequency control. The range of this frequency-control condenser is approximately 3 parts in $10^{6}$, and during this time only half of this total adjustment was required. This means that the piezo oscillator maintained its frequency within 1.5 parts in $10^{6}$, and with the adjustment it was always possible to set the frequency to agreement with the primary standard. The frequency will remain constant within 2 parts in $10^{8}$ for several hours. On December 1,1932 , the piezo oscillator was moved from the temporary building to the permanent transmitting station at Beltsville, Md. The unavoidable vibration encountered in the change caused an air-bubble to form in the mercury column of the outer thermostat, so that it was necessary to readjust this thermostat. At this same time new tubes were again installed. The piezo oscillator has given very satisfactory service in the standard-frequency transmissions.

\section{ACKNOWLEDGEMENT}

F. S. Ringler constructed the piezo oscillator described in this paper, and it is desired to acknowledge the many valuable suggestions which he contributed.

Washington, April 22, 1933. 